\title{
A utilização da energia solar de forma mais inteligente, buscando minimizar recursos e maximizar eficiências, por meio de um protótipo.
}

\author{
Alexandra Silva de Araújo - alexandra.araujo@alunos.unis.edu.br \\ Arthur Valverde Condé - arthur.conde@alunos.unis.edu.br \\ Luiz César Tavares - luiz.tavares1@alunos.unis.edu.br \\ João Vytor de oliveira Cardoso - joão.cardoso3@alunos.unis.edu.br \\ Tiago Bittencourt Nazaré - tiago.nazare@professor.unis.edu.br
}

RESUMO: Sabendo que o Brasil possui um extenso potencial energético em virtude dos níveis de incidência solar, a geração de energia fotovoltaica precisa ser amplamente explorada no país, já que possui estímulos fundamentais para isso.

Nesta linha de pensamento, o presente trabalho baseou-se em um levantamento bibliográfico por meio de conceitos teóricos sobre energia solar fotovoltaica e térmica, além de estudos sobre propagação dessa energia e instrumentos de medição, tendo como objetivo final a utilização da energia solar de forma mais inteligente, buscando maximizar sua eficiência.

A partir desses estudos, definiu-se a confecção de uma estrutura para o acoplamento da placa e posterior instalação do sistema de resfriamento. Assim, a estrutura utilizada como suporte foi adaptada para acondicionar uma placa fotovoltaica, com dimensões de 1960 x 990 mm. A partir desta definição elaborou-se o planejamento e a devida ilustração do projeto do suporte para a placa através de um croqui, que pudesse ser reproduzido por meio de um software de Desenho Computadorizado "Solidworks".

Em virtude do que foi mencionado, o presente trabalho objetiva demonstrar por meio de uma experiência prática a possibilidade de aumentar a eficiência ou rendimento da placa fotovoltaica policristalino através de um resfriamento térmico com a utilização de tubulações para troca de calor com a água.

Palavras-chave: Energia Solar, Fotovoltaica, Eficiência, Rendimento 
ABSTRACT: Knowing that Brazil has an extensive energy potential due to the levels of solar incidence, the generation of photovoltaic energy needs to be widely explored in the country, as it has fundamental stimuli for this.

In this line of thought, the present work was based on a bibliographical survey through theoretical concepts on photovoltaic and thermal solar energy, as well as studies on the propagation of this energy and measurement instruments, with the ultimate objective of using solar energy in a manner smarter, seeking to maximize its efficiency.

Based on these studies, a structure for coupling the plate and subsequent installation of the cooling system was defined. Thus, the structure used as support was adapted to accommodate a photovoltaic plate, with dimensions of $1960 \times 990 \mathrm{~mm}$. Based on this definition, the planning and due illustration of the support project for the plate was elaborated through a sketch, which could be reproduced using a "Solidworks" Computerized Drawing software.

In view of what has been mentioned, the present work aims to demonstrate, through practical experience, the possibility of increasing the efficiency or yield of the polycrystalline photovoltaic plate through thermal cooling with the use of pipes for heat exchange with water.

\section{INTRODUÇÃO}

A energia solar no Brasil vem crescendo a passos largos, pois possui diversos benefícios econômicos e ambientais que impulsionam esta fonte de energia renovável. Ela representa apenas $1,7 \%$ de toda a matriz energética, porém, há um crescimento considerável do número de sistemas fotovoltaicos instalados principalmente nas regiões Sul, Sudeste e Nordeste do país.

Sua utilização vem sendo de forma majoritária em residências, com o objetivo de reduzir a conta de luz, seja por meio da energia térmica com o aquecimento da água ou com a utilização da energia fotovoltaica, gerando a eletricidade.

É indiscutível que o Brasil possui uma gigantesca capacidade de energia solar e, graças aos esforços do governo e da iniciativa privada, o país está cada vez mais próximo de ampliar o seu potencial no mercado de energia fotovoltaica. 
Diante desse cenário dinâmico, este trabalho tem como objetivo desenvolver um protótipo, respeitando as normas para utilização de qualquer fonte de energia elétrica no país, regulamentadas pela ANEEL (Agência Nacional de Energia Elétrica). Tal experimento visa potencializar a eficiência e rendimento de placa fotovoltaica policristalino através de um resfriamento térmico com a utilização de tubulações para troca de calor com a água.

\section{REFERENCIAL TEÓRICO}

O Brasil tem potencial para uso de energia solar muito maior que a maioria dos países europeus, sendo inclusive maior que a Alemanha, conforme se observa na figura 1, sendo este último o país que mais investe nesse tipo de energia (PEREIRA et al., 2017; FERREIRA_et_al.,_2018). Vale ressaltar que a imagem apresentada possui escalas distintas de cores.

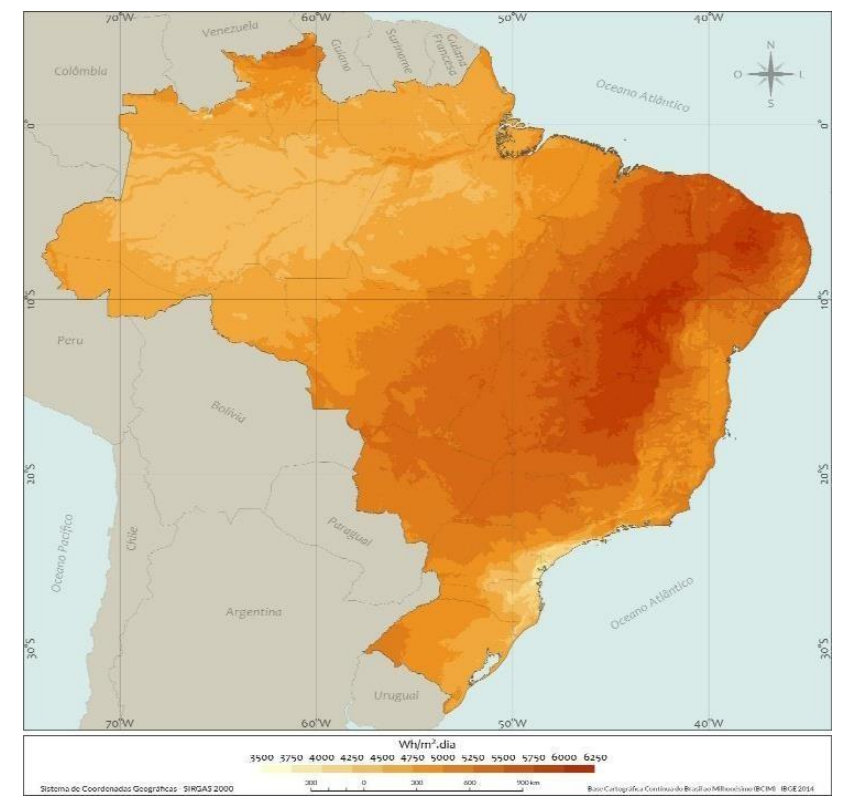

Figura 1 - Média anual do total diário da irradiação no plano inclinado na latitude no Brasil. Fonte: (PEREIRA et al. 2017).

A radiação solar varia de acordo com a estação do ano e a região, sendo de 1500 a $2300 \mathrm{kWh} / \mathrm{m}^{2} / \mathrm{ano}$, enquanto na Alemanha é de 900 a $1250 \mathrm{kWh} / \mathrm{m}^{2} / \mathrm{ano}$. Sua extensa localização entre os trópicos de Câncer e Capricórnio faz com que receba altas taxas de radiação, quase que perpendiculares, aumentando assim seu potencial de geração de energia fotovoltaica. 
Segundo DIAS et al. (2017), a energia fotovoltaica tem recebido mais atenção do governo recentemente. Já existe regulamentação para plantas de microgeração e minigeração de energia produzidas a partir de fontes renováveis, e o Banco Nacional de Desenvolvimento Social e Econômico (BNDES) possui opções atrativas de financiamento para a instalação desse tipo de energia, porém apenas para grandes empresas (SILVA, 2015). Os autores também ressaltam que alguns fatores impeditivos estão sendo removidos a partir de mudanças na legislação brasileira, como redução de impostos para empresas de sistemas de transmissão e distribuição desse tipo de energia e remoção de impostos federais e estaduais incidentes sobre a energia gerada pelo consumidor e entregue à concessionária.

De acordo com (AKINYELE, 2017), o número de habitantes do mundo que não tem acesso à eletricidade moderna é de 200 em cada 1000 pessoas. Este é um dos motivos que levou a Organização das Nações Unidas-ONU, a lançar os Objetivo de Desenvolvimento Sustentável (DPSs), considerando dentre os diversos objetivos o que estimula o fornecimento do acesso à energia limpa e acessível para todos até o ano de 2030, sendo que a geração de energia solar fotovoltaica, oriunda de matriz energética renovável, é uma das opções para atingir tal objetivo.

A geração de energia fotovoltaica é de fato uma alternativa para diversificar a matriz energética com vasta possibilidade de exploração no Brasil, pelas características naturais que este possui. No entanto, apesar de ser um tema cada vez mais discutido, inclusive pela academia, sua implantação efetiva ainda é muito restrita, principalmente pelos altos custos de instalação e pela falta de incentivos governamentais.

Analisando os poucos projetos instalados por meio de ações do governo, verificase que estes utilizam sistemas autônomos e tem foco em casas isoladas, normalmente distantes das redes de distribuição (FERREIRA, KUNH, FAGNANI, DE SOUZA, TONEZER, DOS SANTOS \& COIMBRA - ARAÚJO, 2018).

A placa solar (também chamada de módulo ou painel fotovoltaico) é o equipamento fundamental na produção de energia elétrica fotovoltaica. Como é possível observar na figura 2, o módulo é composto por um conjunto de células fotovoltaicas que são responsáveis por converter a luz solar em eletricidade. 


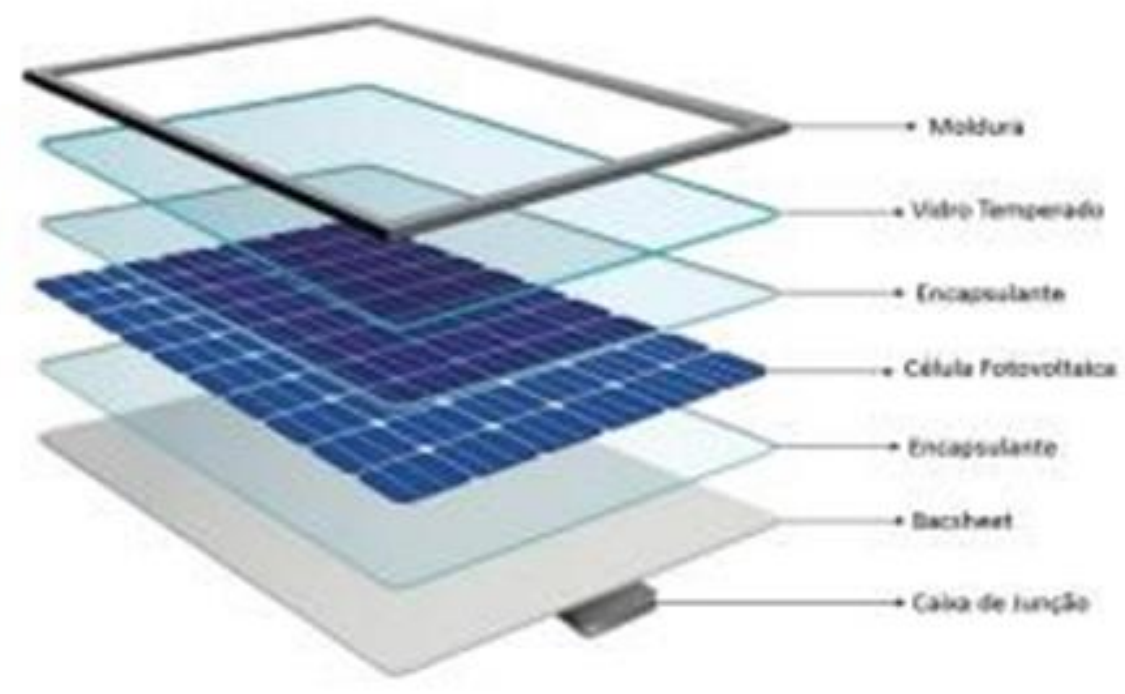

Figura 2 - Estrutura de placa solar fotovoltaica.

Fonte: Blog minha casa solar (2019).

Construção das Placas Solares Semicondutores são materiais intermediários entre condutores e isolantes, possuem baixa condutividade elétrica. Segundo Dias:

\footnotetext{
“Os semicondutores não conseguem conduzir corrente elétrica em condições químicas normais. Os átomos dos semicondutores são tetravalentes, ou seja, possuem apenas quatro camadas de valência, o que os torna elementos não estáveis”. (DIAS, 2019, p.75)
}

De acordo com LUCEÑO-SÁNCHEZ; DÍEZ-PASCUAL; PEÑA CAPILLA, (2019) as células fotovoltaicas são os dispositivos base usados para converter a radiação solar em energia elétrica a partir do efeito fotovoltaico. A arquitetura base das células fotovoltaicas segue o princípio da união de duas regiões semicondutoras com diferentes concentrações de elétrons, e, assim sendo, esses materiais podem ser do tipo n, ou seja, aqueles que possuem excesso de elétrons, ou tipo p, que são semicondutores com um excesso de cargas positivas, todavia, em ambos os casos, o material é eletronicamente neutro. Quando ambas as regiões, p e n, entram em contato a partir do fechamento do circuito elétrico, o excesso de cargas positivas flui da região p e dos elétrons da região $n$ através da junção pn, esse movimento de cargas é chamado de corrente de difusão.

Já os íons fixos próximos à junção pn geram um campo elétrico na direção oposta à difusão, o que gera, também, uma corrente de desvio. Em equilíbrio, a corrente de difusão é balanceada pela corrente de desvio, de modo que a corrente líquida é zero, nessas 
condições, uma barreira potencial é estabelecida na junção pn. À medida que a radiação solar atinge a célula, a contribuição de energia dos fótons é absorvida pelos elétrons que podem romper suas ligações, produzindo, então, pares de elétrons - "holes". Esses portadores de carga são empurrados pelo campo elétrico e conduzidos por meio da junção pn. Se uma carga externa estiver conectada, uma corrente elétrica e uma diferença de potencial entre os terminais da célula serão estabelecidas.

Tipos de Placas com o processo de dopagem é fabricado três tipos de células fotovoltaicas, são elas: célula de silício monocristalino, célula de silício policristalino e célula de silício amorfo.

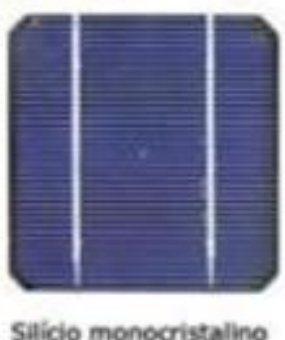

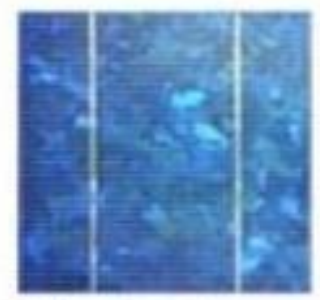

SAlicio policristalino

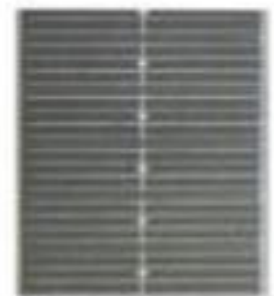

Silicio amorfo

Figura 3 - Tipos de células mais utilizadas. Fonte: Jurinic (2020).

As células de silício monocristalino (mono-Si) contam com uma tecnologia mais antiga se comparada com os outros tipos, porém, sua eficiência na conversão de luz solar em energia elétrica fica entre 14 e $21 \%$, devido sua boa eficiência, podem converter mais energia ocupando menos espaço se comparado com as células policristalinas ou de filme fino. Painéis de silício monocristalino são facilmente reconhecidos pela cor uniforme (geralmente azulado escuro), característica de silício de alta pureza (99,9999\% de pureza) (SILVA, 2016).

Células de silício policristalino (poli-Si) Células de silício policristalinos (poliSi) utilizam a mesma matéria prima que as células monocristalinas, a principal diferença é o modo de fundição dos cristais. No policristalino, a fundição acontece em um bloco, sendo assim, esse método de fundição preserva a formação de múltiplos cristais, quando o cristal é cortado e fatiado é perceptível essa formação múltipla de cristais. Sua eficiência é ligeiramente menor que a do monocristalino, ficando entre 13 e $17 \%$, isso acontece devido à baixa pureza do silício policristalino. Tem como vantagem o custo menor em 
comparação com o monocristalino, por ter um processo de produção menos rigoroso e um baixo grau de pureza (SILVA, 2016).

Células de silício amorfo (a-Si) Nas células de silício amorfo (a-Si), a eficiência é ainda menor (entre 5 e 7\%). Essas células são obtidas a partir da deposição de camadas finas de silício sobre superfícies de vidro ou metal.

Devido à baixa eficiência das células de silício amorfo, sua utilização se retinha a aplicações em pequena escala (por exemplo, calculadoras de bolso), atualmente, com uma técnica de fabricação conhecida como "empilhamento" várias células de silício amorfo podem ser combinadas resultando em taxas mais eficientes de conversão (de 6 a 9\%), permitindo o uso também em larga escala (MAIA, 2016).

\section{METODOLOGIA}

O levantamento bibliográfico apresentou conceitos teóricos sobre energia solar fotovoltaica e térmica, estudos sobre propagação da energia solar e instrumentos de medição além de estudos sobre trocadores de calor, tendo como objetivo a utilização da energia solar de forma mais inteligente, buscando maximizar sua eficiência. Para atendimento do objetivo do trabalho, foi desenvolvido um protótipo composto por uma estrutura de acoplamento da placa e sistema de resfriamento.

A estrutura utilizada como suporte foi pensada para acondicionar uma placa fotovoltaica, cujas dimensões são 1960 x 990 mm. A partir desta definição elaborou-se o planejamento e a devida ilustração do projeto do suporte para a placa através de um croqui, que pudesse ser reproduzido por meio de um software de Desenho Computadorizado "Solidworks", versão (2010, 64bits).

A construção se deu com barras de metalons de aço, perfis e soldados com eletrodo e para o acabamento foi realizada a pintura. A estrutura também prevê um encaixe com uma angulação possibilitando a adequação da superfície da placa de forma perpendicular aos raios solares incidentes. 


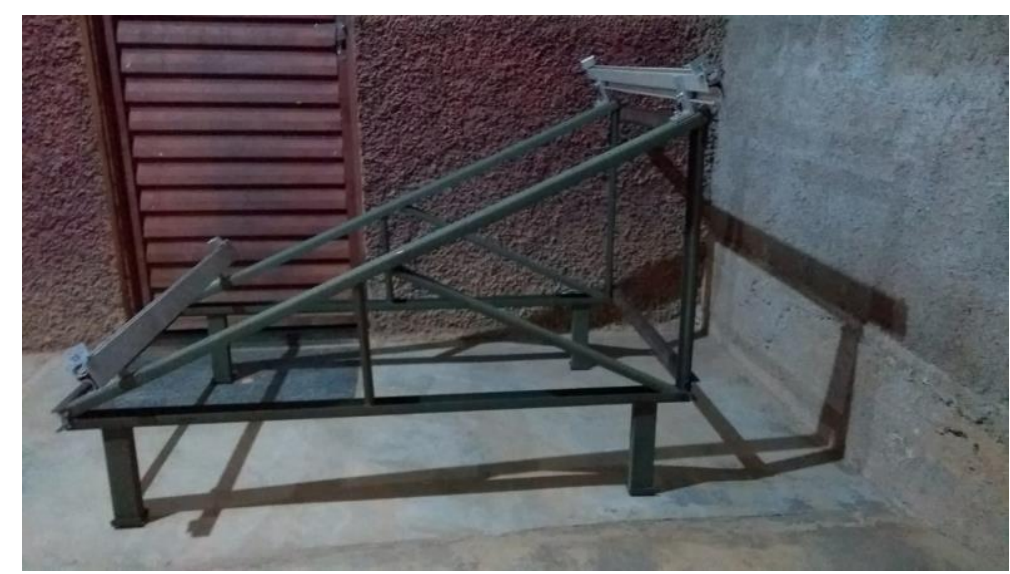

Figura 4 - Suporte para fixação da placa fotovoltaica

Fonte: imagem dos autores.

Para completar o sistema foram colocadas tubulações de cobre, na parte inferior da estrutura, que permitam a circulação de água fria com o objetivo de reduzir a temperatura da placa e aumentar a eficiência e duração do trabalho durante o dia. Para acompanhar a temperatura será utilizado um termômetro digital infravermelho. Além disso, serão utilizados outros materiais essenciais, tais como:

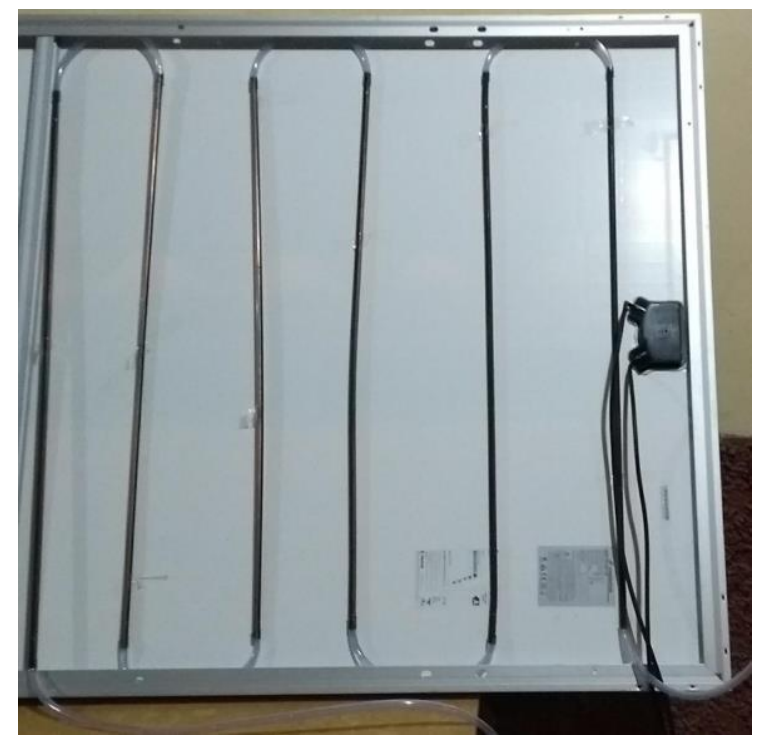

Figura 5 - Sistema de resfriamento adaptado na placa fotovoltaica

Fonte: imagem dos autores.

- Módulos FV das melhores marcas do mercado - TIER 1;

- Especificações da placa fotovoltaica policristalino; 
Os quadros 1 a 4 apresentam dados e características de temperatura da placa fotovoltaica utilizada.

\section{Quadro 1- Dados elétricos | STC *}

\begin{tabular}{|l|l|}
\hline CS3U & $345 \mathrm{P}$ \\
\hline Nominal Max. Power (Pmax) & $345 \mathrm{~W}$ \\
\hline Opt. Operating Voltage (Vmp) & $39.0 \mathrm{~V}$ \\
\hline Opt. Operating Current (Imp) & $8.86 \mathrm{~A}$ \\
\hline Open Circuit Voltage (Voc) & $46.4 \mathrm{~V}$ \\
\hline Short Circuit Current (Isc) & $9.43 \mathrm{~A}$ \\
\hline Module Efficiency & $17.39 \%$ \\
\hline Operating Temperature & $-40^{\circ} \mathrm{C} \sim+85^{\circ} \mathrm{C}$ \\
\hline Max. System Voltage & $1000 \mathrm{~V}(\mathrm{IEC} / \mathrm{UL})$ or \\
& $1500 \mathrm{~V}(\mathrm{IEC} / \mathrm{UL})$ \\
\hline Module Fire Performance & $\mathrm{TYPE} 1$ (UL 1703) or CLASS C \\
& $($ IEC 61730) \\
\hline Max. Series Fuse Rating & $30 \mathrm{~A}$ \\
\hline Application Classification & Class A \\
\hline Application Classification & $0 \sim+5 \mathrm{~W}$ \\
\hline
\end{tabular}

Quadro 2- Dados mecânicos

\begin{tabular}{|l|l|}
\hline Specification & Data \\
\hline Cell Type Poly-crystalline & $156.75 \times 78.38 \mathrm{~mm}$ \\
\hline Cell Arrangement & $144[2 \times(12 \times 6)]$ \\
\hline Dimensions & $\begin{array}{l}2000 \times 992 \times 40 \mathrm{~mm} \\
(78.7 \times 39.1 \times 1.57 \mathrm{in})\end{array}$ \\
\hline Weight & $22.6 \mathrm{~kg}(49.8 \mathrm{lbs})$ \\
\hline Front Cover & $3.2 \mathrm{~mm}$ tempered glass \\
\hline Frame & $\begin{array}{l}\text { Anodized aluminium alloy, } \\
\text { crossbar enhanced }\end{array}$ \\
\hline J-Box & IP68, 3 diodes \\
\hline
\end{tabular}




\begin{tabular}{|l|l|}
\hline Cable & $4.0 \mathrm{~mm}^{2} \& 12$ AWG \\
\hline Cable Length & $1670 \mathrm{~mm}(65.7$ in \\
\hline Connector & T4 (IEC / UL) \\
\hline Per Pallet & 27 pieces \\
\hline Per Container & (40` HQ) 594 pieces \\
\hline
\end{tabular}

\section{Quadro 3- Dados Elétricos | NMOT *}

\begin{tabular}{|l|l|}
\hline CS3U & $345 \mathrm{P}$ \\
\hline Nominal Max. Power (Pmax) & $255 \mathrm{~W}$ \\
\hline Opt. Operating Voltage (Vmp) & $35.6 \mathrm{~V}$ \\
\hline Opt. Operating Current (Imp) & $7.17 \mathrm{~A}$ \\
\hline Open Circuit Voltage (Voc) & $43.3 \mathrm{~V}$ \\
\hline Short Circuit Current (Isc) & $7.61 \mathrm{~A}$ \\
\hline
\end{tabular}

Quadro 4 - Características de temperatura

\begin{tabular}{|l|l|}
\hline Specification & Data \\
\hline Temperature Coefficient (Pmax) & $-0.38 \% /{ }^{\circ} \mathrm{C}$ \\
\hline Temperature Coefficient (Voc) & $-0.29 \% /{ }^{\circ} \mathrm{C}$ \\
\hline Temperature Coefficient (Isc) & $0.05 \% /{ }^{\circ} \mathrm{C}$ \\
\hline Nominal Module Operating Temperature & $43 \pm 2{ }^{\circ} \mathrm{C}$ \\
\hline
\end{tabular}

Fonte: SICES Solar

- Conjunto de cabos solares (preto, vermelho e verde/amarelo) com proteção UV;

- Estrutura para sustentação de Módulos Fotovoltaicos;

- Pares de conectores, modelo: MC4 (Origina);

Durante a ação prática do projeto, o teste inicial foi realizado sem a adaptação de tubulações de cobre na parte de baixo do protótipo e a placa fotovoltaica foi posicionada na direção ao norte $20^{\circ}$, com a inclinação de $25,18^{\circ}$.

A temperatura da placa inteira foi aferida no intervalo de 15 minutos, utilizandose um termômetro infravermelho digital laser industrial (especificações: cor laranja, material: plástico, caixa de medição de temperatura: $-50-380^{\circ} \mathrm{C}$, precisão: $+/-1,5^{\circ} \mathrm{C}$ ou $+/-1,5 \%)$. 
Os testes tiveram início às $10 \mathrm{~h} 05 \mathrm{~min}$, com a temperatura total da placa de $32^{\circ} \mathrm{C}$, aumentando para $47.5^{\circ} \mathrm{C}$ na segunda aferição ocorrida às $10 \mathrm{~h} 20 \mathrm{~min}$.

Todo processo foi repetido às $12 \mathrm{~h} 00 \mathrm{~min}$, com o diferencial da tubulação de cobre adaptada debaixo da placa, utilizando pedaços de mangueira de nível cristal siliconada 5/16" para fazer as ligações entre elas, totalizando 4,5m de tubulação de cobre com espessura $3 / 8$ ".

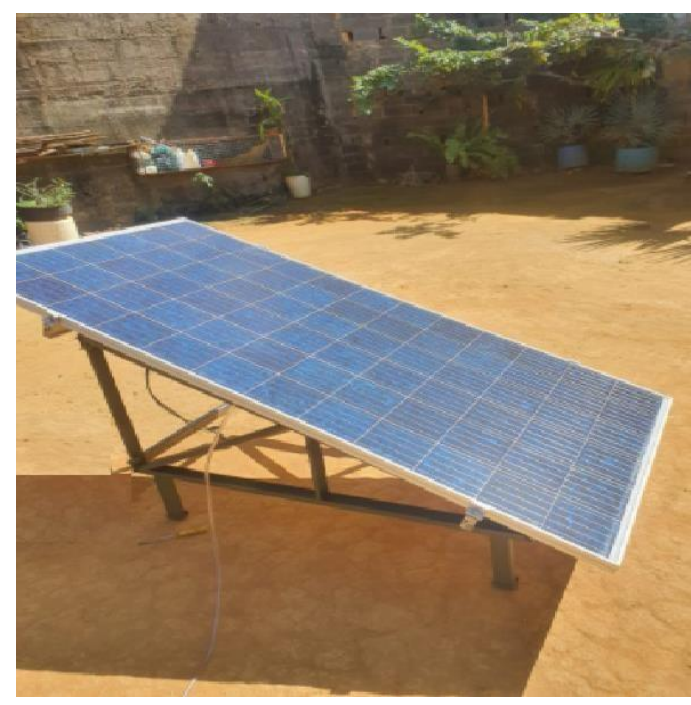

Figura 6 - Protótipo

Fonte: imagem dos autores.

\section{RESULTADOS E DISCUSSÕES}

Os resultados indicaram que a temperatura da placa sem a circulação de água no sistema foi de $53,7^{\circ} \mathrm{C}$, mantendo sua eficiência de $17,75 \%$. Após a abertura da água corrente da concessionária para o protótipo, a água passou a circular com a temperatura de entrada de $21,7^{\circ} \mathrm{C}$ para dentro da tubulação de cobre.

Nesse contexto, a água entrou na parte inferior da placa e saiu da parte superior com uma média de temperatura de $25^{\circ} \mathrm{C}$. Após um minuto, a temperatura da placa foi aferida e apresentou uma redução para $49,3^{\circ} \mathrm{C}$, obtendo-se diminuição na média de temperatura de $4,4^{\circ} \mathrm{C}$. 


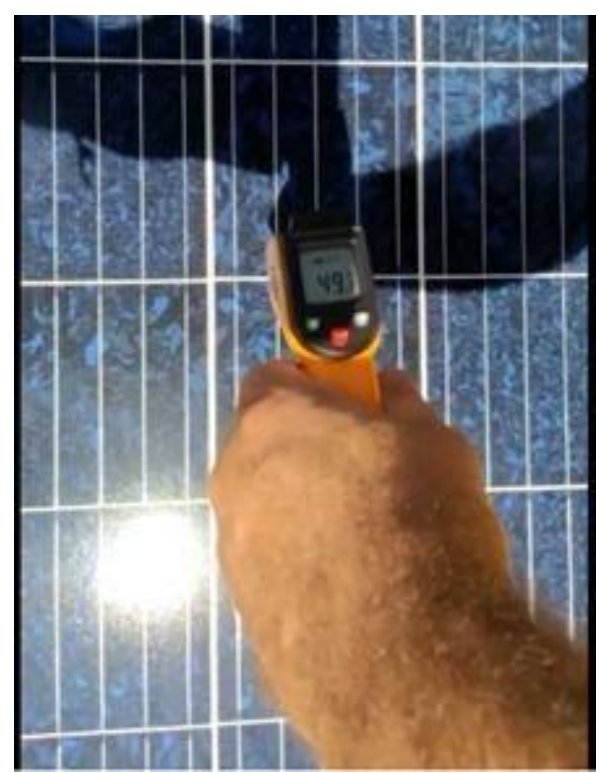

Figura 7 - Medição da temperatura da placa pelo termômetro infravermelho.

Fonte: imagem dos autores.

Considerando essa redução na temperatura, para cada $1^{\circ} \mathrm{C}$ que a placa perde, ganha-se $0,5 \%$ a mais no seu rendimento. Sendo assim, obteve-se maximização de rendimento na geração de energia da placa fotovoltaica policristalino de 2,2 \%. Para melhor exemplificar, uma placa com geração de 2000 watts aumenta o seu rendimento para 2044 watts. Sendo assim, esse ganho de 44 watts equivale a 7 horas de uma lâmpada de 6 watts ligada.

\section{CONCLUSÃO}

Considerando que nosso país possui um extenso potencial energético em virtude dos níveis de incidência solar e que este recurso precisa ser amplamente explorado e estimulado, o presente trabalho objetivou demonstrar a utilização da energia solar de forma mais inteligente, buscando potencializar seu rendimento e sua eficiência.

Levando-se em conta esta observação, realizou-se um levantamento bibliográfico sobre energia solar fotovoltaica e térmica, além de estudos sobre propagação dessa energia e instrumentos de medição para nortear a experiência realizada. 
Nesse enfoque, definiu-se a construção de uma estrutura para o acoplamento da placa e posterior instalação do sistema de resfriamento. Por meio desse sistema, foi possível demonstrar com uma experiência prática a possibilidade de aumentar a eficiência ou rendimento da placa fotovoltaica policristalino através de um resfriamento térmico com a utilização de tubulações para troca de calor com a água.

Durante a ação prática do projeto, foi observada uma redução de $4.4^{\circ} \mathrm{C}$ na temperatura da placa. Em decorrência disso, foi obtido um rendimento de 2,2\% na geração de energia da placa.

O trabalho atingiu seu objetivo na medida em que verificou-se a maximização da eficiência e rendimento de placa fotovoltaica policristalino, por meio de resfriamento térmico com a utilização de tubulações para troca de calor com a água.

Como possíveis trabalhos futuros, pode-se apontar:

(1) O intuito é aumentar as tubulações de cobre em uma metragem 9 a 10 metros, para que possamos diminuir o espaçamento entre um cano ao outro. Com isso iremos ter um fluxo maior de passagem da água em contato com a placa para seu resfriamento.

(2) Aumentar o monitoramento das medições de rendimento da placa fotovoltaica, em diferentes dias, por várias horas.

(3) Substituir a mangueira de nível cristal siliconada 5/16" por uma mangueira preta aquecimento solar de água serpentina $3 / 8$ ".

(4) Fazer um sistema de deposito da água para reaproveitamento utilizando uma boia com intuito de reduzir a saída, aumentando o tempo da água dentro da tubulação de cobre para melhor troca térmica. 


\section{REFERÊNCIAS BIBLIOGRÁFICAS}

COSTA, Raycam Evaristo de oliveira. O uso de placas fotovoltaicas: uma revisão bibliográfica. Monografia apresentada à Universidade Federal Rural do Semi-Árido como requisito para obtenção do título de Bacharel em Ciência e Tecnologia Defendida em: 12/2020. Repositório UFERSA. Disponível em:

https://repositorio.ufersa.edu.br/bitstream/prefix/5962/1/RaycamEOC_MONO.pdf

Acesso em: 29 de março de 2021.

COSTA, Suellen Caroline Silva; ABREU, Amanda Mello Faria Veloso; VIANA, Marcelo Machado; BRITO, Pedro Paiva; ASSIS, Marcelus Vasconcelos de; MAIA, Cristiana Brasil; DINIZ, Antonia Sonia Alves Cardoso; KAZMERSKI Lawrence Lee. Caracterização físico-química da sujidade depositada sobre módulos fotovoltaicos instalados em zonas climáticas de minas gerais. VI Congresso Brasileiro de Energia Solar - Belo Horizonte, 07 de abril de 2016. Disponível em: <.http://www.pvreliability.org/ABENS(Suellen).pdf>Acesso em: 29 de março de 2021.

DIAS, Damiana Pâmella da Silva. Estudo de implantação de um sistema de produção de energia solar através de painéis fotovoltaicos em um hospital. Revista Científica Multidisciplinar Núcleo do Conhecimento. Ano 05, Ed. 05, Vol. 13, pp. 57-75. Maio de 2020. ISSN: 2448-0959. Disponível em: <https://www.nucleodoconhecimento.com.br/engenharia-mecanica/sistema-deproducao> Acesso em: 03 de abril de 2021.

DIAS, Matheus; MARONEZE,Camila. Nanoestruturas plasmõnicas e semicondutores 2D aplicados em processos de conversão de energia solar. Programa institucional de iniciação científica e iniciação tecnológica, 2019-12-04. Disponivel em: <http://eventoscopq.mackenzie.br/index.php/jornada/xvjornada/paper/viewPaper/1676> Acesso em: 29 de março de 2021.

MORAES, Caio. Célula fotovoltaica: Tudo que você precisa saber. Blog Eletrônica de potência , 05/2020 . Disponível em: <https://eletronicadepotencia.com/celulafotovoltaica/> Acesso em: 03 de abril de 2021.

PASCUAL, Ana María Díez; CAPILLA,Rafael Peña; SÁNCHEZ,José Antonio Luceño . Materiais para energia fotovoltaica: o estado da arte e desenvolvimentos recentes. International Journal of Molecular Sciences. 2019; 20 (4): 976. Disponível em: <https://www.mdpi.com/1422-0067/20/4/976> Acesso em: 29 de março de 2021.

PASQUALOTTO, Bruna; LAZZARI, Luciana Salete; BRADALISE, Loreni Teresinha; BERTOLIN, Geysler Rogis Flor. A utilização de placas fotovoltaicas como vantagem competitiva / The use of photovoltaic panels as a competitive advantage. Brazilian journal of developmente, vol 5, no 1,2019. Disponível em: $<$ https://www.brazilianjournals.com/index.php/BRJD/article/view/804> Acesso em:29 de março de 2021. 
PEREIRA, Narlon Xavier. Desafios e perspectivas da energia solar fotovoltaica no Brasil: geração distribuída vs geração centralizada. Repositório Institucional UNESP, 201901-31. Disponível em: 〈http://hdl.handle.net/11449/181288> Acesso em: 03 de abril de 2021.

Principais tipos de células fotovoltaicas constituintes de painéis solares Nov 28, 2017 < https://www.portal-energia.com/principais-tipos-de-celulas-fotovoltaicas-constituintesde-paineis-solares/> Acesso em: 03 de abril de 2021.

SAMPAIO, Leobino Nascimento. Gestão do Desenvolvimento Territorial/Cidades inteligentes. Repositório UFBA, Escola de Administração; Superintendência de Educação a Distância 2020 ISBN: 978-65-5631-010-7. Disponível em: <https://repositorio.ufba.br/ri/bitstream/ri/32559/1/eBook_CI-GDT_Final_c.pdf >Acesso em: 29 de março de 2021.

SILVA, Gardenio Diogo Pimentel da; SOUZA, Marcelo José Raiol. Análise de variáveis de projeto de sistema solar fotovoltaíco utilizando o modelo sam: uma comparação entre Belém, Fortaleza e Brasília. Revista UFPR, 2016. Disponível em: <https://www.semanticscholar.org/paper/AN\%C3\%81LISE-DE-VARI\%C3\%81VEISDE-PROJETO-DE-SISTEMA-SOLAR-O-SilvaSouza/9d29966088e866bb34f9b8ad402804378b9272bc\#citing-papers> Acesso em: 29 de março de 2021. 